\title{
Was macht glücklich?
}

Eine der wichtigsten Aufgaben der Glücksforschung besteht im Bestimmen, Isolieren und Messen der verschiedenen Einflussgrößen auf das Glück.

Die Daten zur subjektiven Lebenszufriedenheit einzelner Personen werden mithilfe multipler Regressionen mit (möglichen) Bestimmungsfaktoren des Glücks miteinander in Beziehung gesetzt. Dabei werden sowohl einfache wie auch weiter fortgeschrittene nicht-lineare statistische Verfahren verwendet. Entscheidend ist, dass gleichzeitig der Einfluss vieler unterschiedlicher Faktoren berücksichtigt wird. In manchen ökonometrischen Schätzungen werden simultan 20 bis 30 Variablen einbezogen. Dieses Vorgehen erlaubt, den Einfluss einzelner Bestimmungsgründe zu erfassen, wobei der Einfluss aller anderen Bestimmungsgründe konstant gehalten werden kann. Wird etwa untersucht, welchen Einfluss das Einkommen auf die subjektive Lebenszufriedenheit hat, werden das Alter, der Zivilstand, die Religion und viele andere Variablen konstant gehalten. Die abgeleiteten Aussagen beziehen sich immer auf einen Durchschnitt. Es ist somit stets möglich, dass eine einzelne Person davon abweicht.

In den folgenden Teilen wird ein kurzer Überblick über die Bestimmungsgründe des Glücks gegeben:

- Genetische Ausstattung

- Wirtschaftliche Faktoren

- Soziodemografische Einflüsse

- Kultur und Religion

- Glück und Politik. 